\title{
Chemistry and Materials Science Directorate 2002 Postdoctoral Symposium
}

\author{
B.D. Wirth
}

August 14, 2002

U.S. Department of Energy

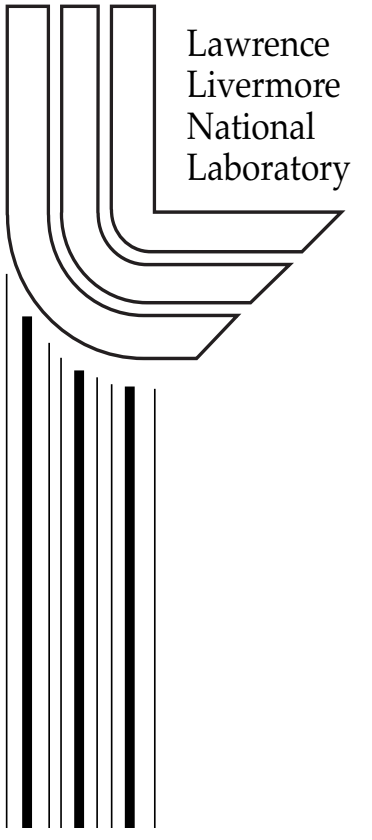




\section{DISCLAIMER}

This document was prepared as an account of work sponsored by an agency of the United States Government. Neither the United States Government nor the University of California nor any of their employees, makes any warranty, express or implied, or assumes any legal liability or responsibility for the accuracy, completeness, or usefulness of any information, apparatus, product, or process disclosed, or represents that its use would not infringe privately owned rights. Reference herein to any specific commercial product, process, or service by trade name, trademark, manufacturer, or otherwise, does not necessarily constitute or imply its endorsement, recommendation, or favoring by the United States Government or the University of California. The views and opinions of authors expressed herein do not necessarily state or reflect those of the United States Government or the University of California, and shall not be used for advertising or product endorsement purposes.

This work was performed under the auspices of the U. S. Department of Energy by the University of California, Lawrence Livermore National Laboratory under Contract No. W-7405-Eng-48.

This report has been reproduced directly from the best available copy.

Available electronically at http://www.doc.gov/bridge

Available for a processing fee to U.S. Department of Energy

And its contractors in paper from

U.S. Department of Energy

Office of Scientific and Technical Information

P.O. Box 62

Oak Ridge, TN 37831-0062

Telephone: (865) 576-8401

Facsimile: (865) 576-5728

E-mail: reports@adonis.osti.gov

Available for sale to the public from

U.S. Department of Commerce

National Technical Information Service

5285 Port Royal Road

Springfield, VA 22161

Telephone: (800) 553-6847

Facsimile: (703) 605-6900

E-mail: orders@ntis.fedworld.gov

Online ordering: http:/ / www.ntis.gov/ordering.htm

\section{OR}

Lawrence Livermore National Laboratory

Technical Information Department's Digital Library

http: / / www.llnl.gov/tid/Library.html 
Chemistry and Materials Science Directorate

\section{Postdoctoral Symposium \\ August 22, 2002 \\ B235, Gold Room}
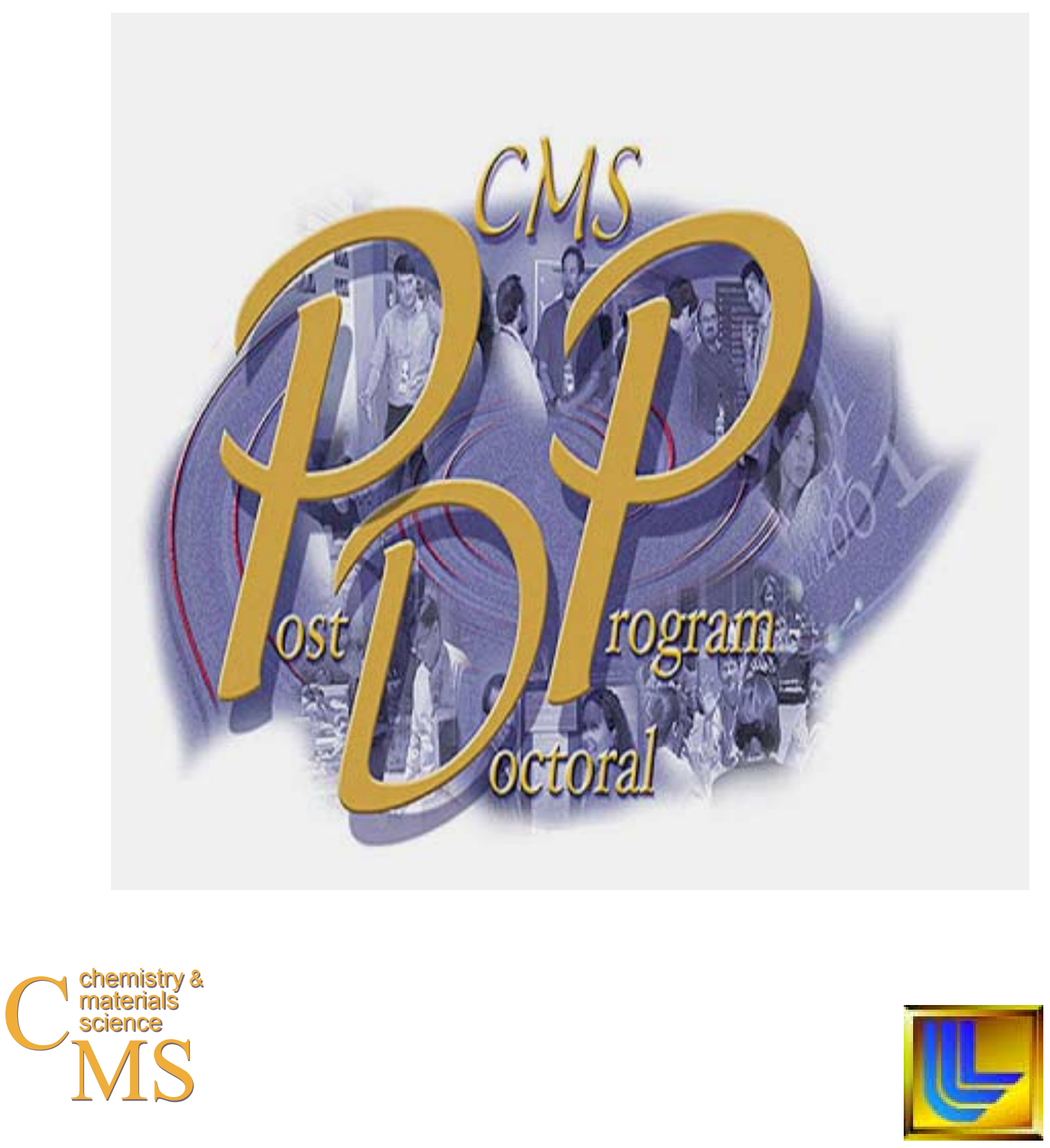


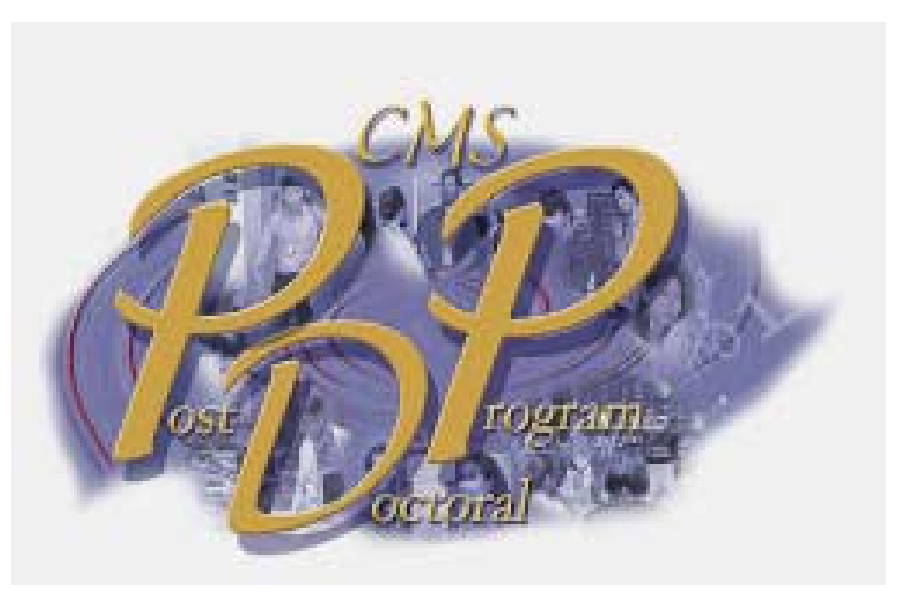

Brian D. Wirth

Director, CMS Postdoctoral Program

Ph: (925) 424-9822, Fax: (925) 423-7040

wirth4@1lnl.gov

Athanasios (Tom) Arsenlis

Deputy Director, CMS Postdoctoral Program

Ph: (925) 424-2584, Fax: 424-5205

arsenlis1@1lnl.gov

\section{CMS Postdoctoral Program Committee}

Anthony (Tony) Esposito (Post-Doc Liaison) (925) 424-3797

Robert Maxwell (ANCD) (925) 423-4491

Joe Zaug (CChED) (925) 423-4428

Maria J. Caturla (MSTD) (925) 422-8964

Chris Orme (MSTD) (925) 423-9509

\section{Administrative Contact:}

Linda Jones

Ph: (925) 423-8839, Fax: (925) 422-7300

This work was performed under the auspices of the U. S. Department of Energy by the University of California, Lawrence Livermore National Laboratory under Contract No. W-7405-Eng-48. 


\section{Chemistry and Materials Science Post-Doctoral Program Symposium Thursday, 22 August 2002 \\ B235, Gold Room}

9:00 Welcome and introductions

Brian Wirth, CMS PD Program

9:05

Roger Qiu, "Study of bio-modulation of calcium oxalate crystallization by in situ atomic force microscopy"

9:25 Jenny Heidbrink, "Structural characterization of non-covalent protein/DNA interactions by using mass spectrometry"

9:45 Chad Talley, "Surface enhanced Raman spectroscopy on biological systems"

10:05 Olgica Bakajin, "Single molecule measurements of protein folding kinetics"

10:25 Break, poster session

11:00 Louisa Hope-Weeks, "Preparation of functionalised silicon nanocrystals via a solution route"

11:20 Wei Cai, “A Mechanism for Anomalous Dislocation Multiplication in FCC Metals"

11:40 Kurt Glaesemann, "Quantum vibrations in molecules"

12:00 Lunch

1:00 Poster Session

2:30 Mitchell Anthamatten, "Controlling surface roughness in polyimide NIF targets by solvent-vapor exposure"

2:50 David Fergenson, "Real-time detection of biological aerosols using bioaerosol mass spectrometry"

3:10 Kevin Vandersall, "Experimental and modeling studies of crush, puncture, and perforation scenarios in the Steven impact test"

3:30 Jonathan Crowhurst, "Impulsive stimulated light scattering in the diamond anvil cell"

3:50 Award presentations and wrapup

4:00 Adjourn 


\section{POSTERS}

Kerri Blobaum "Investigating the $\alpha$ ' $/ \delta$ Martensitic Phase Transformation in Pu-Ga Alloys"

Eduardo Bringa "Simulating shock-induced plasticity in single crystal Cu"

Julio Camarero "Using segmental isotopic labeling and NMR spectroscopy for studying the mechanism of bacterial transcription. A potential target for the design of new antibiotics"

Sarah Chinn

"Advances in High Sensitivity Portable LC/NMR"

Barry Cheung

"Assembly of oriented 2-dimensional protein and virus arrays by dip-pen nanolithography"

Wonyoung Choe

"Structure and bonding of magnetocaloric materials $\mathrm{Gd}_{5}\left(\mathrm{Si}_{\mathrm{x}} \mathrm{Ge}_{1-\mathrm{x}}\right)_{4}$ and Related Systems"

Brady Clapsaddle "The Sol-gel Synthesis and Characterization of $\mathrm{Fe}_{2} \mathrm{O}_{3}-\mathrm{SiO}_{2}$ Nanocomposite Materials with a Large Iron Oxide Content"

Anthony Esposito "Using Micro-Raman Spectroscopy for Rapid Identification of Bacterial Spores”

Stephen Glade "Characterization of Nanostructural Features in Reactor Pressure Vessel Model Alloys"

Bradley Hart

"Development of an Enzyme-Based Photoluminescent Porous Silicon Detector"

Andrea Hodge "Synthesis of Nickel-Aluminide Foams"

Tina Jayaweera

"Fate and Transport of Chemical Plumes in the Atmosphere"

Sonia Letant

"Smart membranes: filtration, immobilization and detection on a single chip"

Simon Morton

"Modification of the Spin Polarization, Magnetic Properties and Electronic Structure of Thin Epitaxial Fe Films on GaAs(100) via the Introduction of Substrate Steps"

Erik Nelson

"Multi-Scale Structural Study of the $\delta \rightarrow \alpha$ ' Transformation in a Pu-Ga Alloy"

Jess Sturgeon

"Simulating Nucleation in Irradiated Materials and Aerosols" 
Herbert Tobias "Sampling and analysis of bacterial spores and vegetative bacteria cells using real-time bio-aerosol mass spectrometry"

Ben Torralva

"Electronic and Structural Responses of Materials to Ultashort and Intense Laser Pulses"

Tom Trelenberg "Formation of GaAs Nanocrystalline Films by Femtosecond Pulsed Laser Deposition"

Brandon Weeks "Fabrication of luminescent nanostructures by dip-pen nanolithography"

Alexander Ziegler

"Atomic-Resolution Transmission Electron Microscopy of the Intergranular Structure of a $\mathrm{Y}_{2} \mathrm{O}_{3}$-Silicon Nitride Ceramic" 


\title{
Study of bio-modulation of calcium oxalate crystallization by In Situ Atomic Force Microscopy
}

\author{
S.R. Qiu ${ }^{1}$, C.A. Orme ${ }^{1}$, A. Wierzbicki ${ }^{2}$, J.R. Hoyer ${ }_{5}^{3}$, A.M. Cody ${ }^{4}$, R.D. $\mathrm{Cody}^{4}$, George \\ Nancollas $^{5}$, J.J. De Yoreo ${ }^{1}$ \\ ${ }^{1}$ Department of Chemistry and Materials Science, Lawrence Livermore National Laboratory \\ ${ }^{2}$ Department of Chemistry, University of South Alabama \\ ${ }^{3}$ The Children's Hospital of Philadelphia, University of Pennsylvania \\ ${ }^{4}$ Department of Geological and Atmospheric Sciences, Iowa State University \\ ${ }^{5}$ Department of Chemistry, SUNY Buffalo
}

The understanding of the physical mechanisms by which important biological inhibitors control the nucleation, growth, aggregation, and phase transformation of calcium oxalate crystals at fundamental level is of importance not only to the advances in biomineralization but also to the development of stone disease therapy. Of the three phases of calcium oxalate crystalline, calcium oxalate monohydrate (COM) and dehydrate (COD) are found in the majority of stones formed in the urinary system. Only COM, a major inorganic component of kidney stones, produces adverse physiological effects to human, however. Although a great deal of research has been carried out on the modulation of nucleation, growth, aggregation, and phase transformation of calcium oxalates by biological molecules, the basic mechanism has not yet been determined due to inherent limitations of those techniques that have been utilized

The invention of atomic force microscopy (AFM) has opened a new avenue for the study of the crystal growth in general. One can now probe the growth kinetics and dynamics, and morphology of crystal surfaces down to molecular levels as a typical AFM has a lateral resolution of nanometers. In this study, in situ AFM was used to monitor the COM surface under controlled growth conditions. The growth on both (-1 01$)$ and (010) faces was investigated. The effect of the macromolecules such as citrate and uropontin to the growth of surfaces was also explored. In this presentation, the structural basis for the observed results will be discussed and the implications of the findings to the field of medicine will also be addressed.

This work was performed under the auspices of the U. S. Department of Energy by the University of California, Lawrence Livermore National Laboratory under Contract No. W-7405-Eng-48 and also performed under the auspices of the U.S. National Institutes of Health. 


\title{
Structural Characterization of Non-Covalent Protein/DNA Interactions by Using Mass Spectrometry
}

\author{
Jenny L. Heidbrink, ${ }^{1}$ Michele H. Corzett, ${ }^{2}$ Monique Cosman, ${ }^{2}$ \\ Rod Balhorn, ${ }^{2}$ Sharon J. Shields ${ }^{1}$
}

${ }^{1}$ Chemistry and Materials Science, ${ }^{2}$ Biology and Biotechnology Research Program, Lawrence Livermore National Laboratory, 7000 East Ave., L-231, Livermore CA 94551

Genotoxic mutagens and carcinogens damage DNA via formation of covalently bound DNA adducts. Several biological mechanisms may transpire upon DNA damage, including DNA repair by proteins or mutagenesis that could lead to heritable mutations, cancer or cell death. The DNA repair protein XPA is thought to be essential in recognizing DNA damage and recruiting other proteins to the repair site. Thus far, NMR data has characterized the structure of the complex of damaged DNA with the minimal binding domain of XPA [1], but not the protein/DNA interaction that occurs with the full-length protein. In order to obtain protein function information by elucidating the structure of the XPA/DNA complex, we are developing gas-phase mass spectrometric methods to characterize solution based noncovalent interactions.

ESI- and MALDI-TOF-MS were initially employed to examine the non-covalent binding of a DNA adduct with XPA. Initial studies were carried out with single and double-stranded 11-mer DNA benzo[a]pyrene (BP) adducts and a full-length His-tagged XPA obtained from cell expression. Four stereoisomeric DNA adducts can be obtained from cis and trans addition to deoxyguanosine by the enantiomeric (+)- and (-) -anti-BPDEs (benzo[a]pyrene diol epoxides, the reactive form of metabolized BP). Biological activity and DNA conformations are affected by this stereochemistry. MALDI MS was used to determine that all of the duplex DNA samples contained both of the complimentary strands.

The first sample of XPA supplied $(\sim 1 \mathrm{mg} / \mathrm{mL})$ showed no evidence of the intact protein by MALDI MS. A tryptic digest was performed on this sample, and a peptide map of XPA covering approximately $27 \%$ of the amino acid sequence was obtained. The next sample of XPA was concentrated to $2 \mathrm{mg} / \mathrm{mL}$ in $1 \mathrm{M} \mathrm{NaCl}$ and $50 \mathrm{mM}$ Tris. While this sample exhibited evidence of intact protein, it also showed significant degradation. Reversed phase HPLC was used to purify the XPA from the degradation products, but we could not completely purify XPA at $34 \mathrm{kDa}$. The next batch of XPA was not concentrated, but dialyzed against $100 \mathrm{mM} \mathrm{NH} \mathrm{NH}_{4}\left(\mathrm{HCO}_{3}\right)$ and then lyophilized. Initial reconstitution in $100 \mathrm{mM}$ $\mathrm{NH}_{4}\left(\mathrm{HCO}_{3}\right)$ yielded very little ion signal corresponding to XPA. Yet, proteolysis with trypsin and mass spectrometric analysis of this sample showed that peptides corresponding to XPA digestion were present. These results and others led to questions about the structure, stability and solubility of XPA and what conditions are necessary to study DNA binding by MS.

HPLC studies of the solubility of lyophilized XPA samples in various buffers show that it is only slightly soluble in solvents that are optimal for protein digestion and mass spectrometric analysis. From this study and further results, additional questions regarding DNA binding conditions and sample preparation methods have been raised. Further studies will be carried out to determine the best environment to be used in performing our binding experiments. Once conditions are optimized, we will continue with our original plan to map the protein-DNA binding interface. Eventually, we may be able to compare these results to limited proteolysis combined with gel electrophoresis data, which shows the binding interface to consist of amino acids Met98-Phe219 of XPA [2].

This work was performed under the auspices of the U.S. Department of Energy by University of California Lawrence Livermore National Laboratory under contract No. W-7405-ENG-48.

1. Buchko, G. W.; Tung, C.-S.; McAteer, K.; Isern, N. G.; Spicer, L. D.; Kennedy, M. A. Nucleic Acids Res. 2001, 29, 2635-2643.

2. Kuraoka, I.; Morita, E. H.; Saijo, M.; Matsuda, T.; Morikawa, K.; Shirakawa, M.; Tanaka, K. Mutat. Res. 1996, 362, 87-95. 


\title{
Surface-Enhanced Raman Spectroscopy on Biological Systems
}

Chad E. Talley, Thomas Huser, Christopher W. Hollars and Stephen M. Lane

\author{
Department of Chemistry and Materials Sciences \\ Material Science and Technology Division \\ Lawrence Livermore National Laboratory \\ Livermore, CA 94550
}

Surface-enhanced Raman spectroscopy (SERS) using single metal nanoparticles adsorbed onto a solid support provides a unique tool for investigating biological systems. Biological molecules such as amino acids, nucleic acids, DNA and proteins are attached to the metal nanoparticles using thiol chemistry to confine the molecules to the surface in a well-defined orientation. By carefully selecting the molecular orientation with respect to the nanoparticle surface, specific interactions, such as protein conformational changes and protein-protein association, can be monitored through their Raman spectra. Additionally, dynamics on the millisecond timescale are characterized using a single channel photodetector to monitor distinct Raman frequencies that correspond to specific molecular orientations or binding events. Results from these studies as well as progress toward extending these studies to the single molecule level will be presented.

This work was performed under the auspices of the U. S. Department of Energy by the University of California, Lawrence Livermore National Laboratory under Contract No. W-7405-Eng-48. 


\title{
Single Molecule Measurements of Protein Folding Kinetics
}

\author{
Olgica Bakajin, LLNL \\ E.A. Lipman, B.Schuler and W. A. Eaton, NIH
}

Single molecule techniques have become an important new tool for investigation of the behavior of protein folding reactions. Single molecule Forster resonance energy transfer in freely diffusing proteins can be used to separately determine intramolecular distances in folded and unfolded subpopulations. In order to overcome the limitations of stationary samples, we have combined single molecule fluorescence detection with a microfabricated laminar flow mixer. This allows us to rapidly change the solution conditions and thus directly observe the temporal evolution of the distance distribution as the sample re-equilibrates. We show that single molecule folding kinetics of a small cold shock protein obtained this way are in excellent agreement with ensemble stopped-flow measurements. Our results indicate that this technique has the potential to aid the investigation of more complex reactions and separately resolve processes such as protein collapse and folding. In addition, the technique has great potential for studying other reactions such as DNA-protein interactions on the single molecule level.

This work was performed under the auspices of the U. S. Department of Energy by the University of California, Lawrence Livermore National Laboratory under Contract No. W-7405-Eng-48. 


\title{
Preparation of functionalised silicon nanocrystals via a solution route
}

\author{
Louisa J. Hope-Weeks
}

\section{Lawrence Livermore National Lab, Livermore, CA 94551}

For the last decade silicon nanocrystals have been prepared in solution under a variety of reaction conditions. Each of these methods will be discussed and evaluated to give a brief overview of the subject area. Results obtained from silicon nanocrystals prepared by the reaction of $\mathrm{Mg}_{2} \mathrm{Si}$ with $\mathrm{SiCl}_{4}$ in the presence of an amine solvent, will be presented. The surfaces of the nanocrystals have been passivated with a variety of organic ligands, which range from conjugated systems to simple hydrocarbon chains. FTIR, ${ }^{1} \mathrm{H}$ NMR, ${ }^{13} \mathrm{C}$ NMR, PL (photoluminescence), and PLE (photoluminescence excitation) spectroscopies were used to characterize the nanocrystaline samples. Currently work is underway to investigate conditions under which nanocrystals can be produced with a limited size distribution, in maximum yield. This work has to date involved utilizing and combining in addition to improving several methods previously reported in the literature.

This work was performed under the auspices of the U. S. Department of Energy by the University of California, Lawrence Livermore National Laboratory under Contract No. W-7405-Eng-48. 


\title{
A Mechanism for Anomalous Dislocation Multiplication in FCC Metals
}

\author{
Wei Cai, Maurice de Koning and Vasily V. Bulatov \\ Lawrence Livermore National Laboratory
}

By atomistic simulations, we find that the Frank-Read source for dislocation multiplication can operate anomalously in FCC metals, in that a source of one Burgers vector can repeatedly generate dislocation loops of a different Burgers vector. The mechanism of this behavior is traced to dislocation loop nucleation within the stacking fault of dissociated dislocations. A detailed elasticity model taking into account of the general stacking fault surface is constructed to model the limiting situation, in which an infinitely long perfect dislocation is separated by external stress into two perfect dislocations. Satisfactory agreement is found between this model and direct atomistic simulations. A simplified version of this model is combined with Dislocation Dynamics simulations to examine a wide range of stress conditions, source geometries and materials properties that are beyond the reach of direct atomistic simulations. The conditions for this mechanism to operate are identified, and are found to be readily satisfied in shock experiments. Implications of this mechanism on the interpretation of several recent large-scale atomistic simulations are discussed.

This work was performed under the auspices of the U. S. Department of Energy by the University of California, Lawrence Livermore National Laboratory under Contract No. W-7405-Eng-48. 


\section{Quantum Vibrations in Molecules}

\section{Kurt R. Glaesemann and Laurence E. Fried}

Although the vibration of a molecule is a quantum mechanical phenomenon, the current treatment of molecular vibrations in quantum chemistry is usually based upon the harmonic oscillator approximation. However, wide classes of molecules do not behave as simple harmonic oscillators. To directly take these anharmonic quantum effects into account we have combined a quantum treatment of the electrons via ab initio methods with a quantum treatment of the nuclei via path integral Monte Carlo (PIMC). We have found that this high level of accuracy is needed for generating accurate thermochemical data, such as enthalpies and free energies. This thermochemical data is essential for the calculation of thermochemical rates. To calculate these properties, we developed an efficient parallel PIMC code that achieved substantial computational speedups with new energy estimators and advanced data caching schemes. In the future, we plan to study larger chemical systems in order to gain insight into the molecular structure of biologically relevant molecules. Molecules in which proton transfer is significant are a prototypical example, because the interplay between electronic effects and anharmonic quantum vibrations can be very strong. The fully quantum picture will provide new and exciting insight into these problems that otherwise would be unattainable.

This work was performed under the auspices of the U.S. Department of Energy by the University of California, Lawrence Livermore National Laboratory under Contract No. W-7405-Eng-48. Funding was provided by Laboratory Directed Research and Development (LDRD) Program Grant 02-LW-022. 


\title{
CONTROLLING SURFACE ROUGHNESS IN POLYIMIDE NIF TARGETS BY SOLVENT-VAPOR EXPOSURE
}

\author{
Mitchell Anthamatten, Steve A. Letts, Steve R. Buckley, Chris Chancellor, April E.
} Nissen, Evelyn Fearon, Robert C. Cook

Ultra-smooth polymer surfaces are of great importance in a large body of technical applications, e.g. optical coatings, supermirrors, waveguides, paints, and fusion targets. We are investigating a simple approach to obtaining such surfaces: by temporarily swelling the polymer with solvent molecules. As the solvent penetrates into the polymer, its viscosity is lowered, and surface tension forces drive surface flattening. To investigate sorption kinetics and surface-smoothing phenomena, a series of vapordeposited poly(amic acid) (PAA) films were exposed to dimethyl sulfoxide (DMSO) vapors. During solvent exposure, the surface topology was continuously monitored using light interference microscopy. The resulting power spectra indicate that high frequency defects smooth faster than low frequency defects. This frequency dependence was further investigated by depositing PAA onto a series of 2D sinusoidal surfaces and performing smoothing experiments. The results of our analysis indicate that solventvapor smoothing can be explained using a simple viscous flow model in the framework of a Mullin's analysis.

This work was performed under the auspices of the U.S. Department of Energy by the University of California Lawrence Livermore National Laboratory under contract No. W-7405-Eng-48. 


\section{Real-Time Detection of Biological Aerosols Using Bio-Aerosol Mass Spectrometry}

David P. Fergenson, Herbert J. Tobias, Matthias Frank, Joanne Horne, Paul Steel, Maurice Pitesky and Eric E. Gard

Lawrence Livermore National Laboratory, Livermore, CA 94550, USA

At the Lawrence Livermore National Laboratory, we are developing Bio-Aerosol Mass Spectrometry (BAMS), a system for the real-time analysis and identification of biological aerosols. The BAMS system samples particles directly out of ambient air and analyzes them without any sample preparation. A particle entering the system encounters two scattering laser beams that measure the velocity (and thus the aerodynamic size) of the particle before it reaches the ion source region of the mass spectrometer. A high power pulsed laser is used to desorb and ionize molecules from the incoming particles. Time-of-flight spectra of both positive and negative ions are recorded for each particle together with its size. Our on-line data analysis system recognizes characteristic peaks in the two mass spectra obtained for a single particle and can thus classify the particle.

Our BAMS system was used to measure mass signatures from bacillus and fungal spores in the presence of natural and anthropogenic background materials. We found the spore mass spectra to be quite distinct from each other and from all other non-biological materials we measured. Mass spectra characteristic of each type of material were incorporated into our automated data analysis and particle classification system. As a result, our BAMS system in its present configuration is capable of distinguishing specific biological aerosols including bacillus and fungal spores from other aerosol particles in real time on a particle-by-particle basis.

Here we present recent experimental results from a study in which our BAMS system successfully measured mass signatures from biological aerosols and potential background materials. We discuss the present capabilities of our system in rapidly discriminating between specific biological and non-biological aerosols.

This work was performed under the auspices of the U. S. Department of Energy by the University of California, Lawrence Livermore National Laboratory under Contract No. W-7405-Eng-48. 


\title{
EXPERIMENTAL AND MODELING STUDIES OF CRUSH, PUNCTURE, AND PERFORATION SCENARIOS IN THE STEVEN IMPACT TEST
}

\author{
Kevin S. Vandersall, Steven K. Chidester, Jerry W. Forbes, Frank Garcia, \\ Daniel W. Greenwood, Lori L. Switzer, and Craig M. Tarver \\ Lawrence Livermore National Laboratory \\ Livermore, CA 94550
}

The Steven test and associated modeling has greatly increased the fundamental knowledge of practical predictions of impact safety hazards for confined and unconfined explosive charges. Building on a database of initial work, experimental and modeling studies of crush, puncture, and perforation scenarios were investigated using the Steven impact test. The descriptions of crush, puncture, and perforation arose from safety scenarios represented by projectile designs that "crush" the energetic material or either "puncture" with a pinpoint nose or "perforate" the front cover with a transportation hook. As desired, these scenarios offer different aspects of the known mechanisms that control ignition: friction, shear and strain. Studies of aged and previously damaged HMX-based high explosives included the use of embedded carbon foil and carbon resistor gauges, high-speed cameras, and blast wave gauges to determine the pressure histories, time required for an explosive reaction, and the relative violence of those reactions, respectively. Various ignition processes were modeled as the initial reaction rate expression in the Ignition and Growth reaction rate equations. Good agreement with measured threshold velocities, pressure histories, and times to reaction was calculated for LX-04 impacted by several projectile geometries using a compression dependent ignition term and an elastic-plastic model with a reasonable yield strength for impact strain rates.

This work was performed under the auspices of the U. S. Department of Energy by the University of California, Lawrence Livermore National Laboratory under Contract No. W-7405-Eng-48. 


\title{
Impulsive Stimulated Light Scattering in the Diamond Anvil Cell
}

\author{
Jonathan Crowhurst \\ Lawrence Livermore National Laboratory
}

Experimental methods that are based on the scattering of light from dynamic acoustic excitations have historically proven to be the most direct and effective means for the determination of elastic properties under conditions of ultra-high static pressure. They include the techniques of Brillouin scattering, in which a natural population of thermal phonons is probed, and that of impulsive stimulated light scattering (ISLS), in which the excitations in question are artificially generated. Such methods are appropriate to the high-pressure regime since they do not require mechanical contact with the sample and may also be applied to specimens of near-microscopic dimensions.

Under conditions of high pressure, ISLS has been limited to the study of transparent materials, examples of which include oxygen, water, methanol, and various silicate minerals. Here we report its use to measure the velocity, as a function of pressure, of surface or "interfacial" waves supported at the interface formed by various opaque samples in contact with transparent pressure-transmitting media. The dependence of velocity on direction of these waves has been determined for combinations of single crystal Ge, Ta [2], and Fe in contact with the pressure media liquid methanol, and solid Ar, respectively, and to a maximum pressure of $11 \mathrm{GPa}$. Measurements have also been performed on polycrystalline Ta in an Ar medium, in this case to a pressure of $30 \mathrm{GPa}$. In all cases, calculated velocities, obtained by evaluating the appropriate component of the elastodynamic Green's function, agree closely with measured velocities. Using literature data for the bulk modulus of the opaque samples, and the elastic constants and density of the pressure media, we have been able to obtain the pressure derivatives of some, or all, of the elements of the elastic tensor.

Also to be discussed is an ISLS laboratory recently completed at LLNL. This system has been specifically designed to study opaque materials at high pressure, and will represent a significant evolution of the basic technique.

This work was performed under the auspices of the U. S. Department of Energy by the University of California, Lawrence Livermore National Laboratory under Contract No. W-7405-Eng-48. 


\title{
Investigating the $\alpha^{\prime} / \delta$ Martensitic Phase Transformation in Pu-Ga Alloys
}

\author{
Kerri Blobaum, Jeff Haslam, April Brough, Mark Wall, Adam Schwartz
}

The $\alpha^{\prime} / \delta$ martensitic phase transformation in $\mathrm{Pu}-\mathrm{Ga}$ alloys is unusual and is not well understood. This transformation exhibits a large temperature hysteresis and the maximum amount of $\alpha^{\prime}$ formed is approximately $30 \%$. Here, we report the development of experimental techniques for investigating the stability and transformation of $\mathrm{Pu}-\mathrm{Ga}$ alloys. Because the resistivities of the $\alpha^{\prime}$ and $\delta$ phases differ significantly, four-point probe resistometry is an effective and sensitive technique for monitoring the transformation. Initial resistometry results from thermal cycling of a 2 at $\% \mathrm{Pu}-\mathrm{Ga}$ alloy demonstrate a reproducible transformation hysteresis. The $\alpha^{\prime}$ phase begins to form at 160 $\mathrm{K}$ and reversion to $\delta$ begins at $320 \mathrm{~K}$. Isothermal quench experiments suggest that, at some temperatures, there may be an "incubation period" before $\alpha$ ' begins to form. Differential scanning calorimetry (DSC)will soon be used to further investigate the thermodynamics and kinetics of the transformation. Initial DSC results from a martensitic transformation in a shape-memory alloy are discussed.

This work was performed under the auspices of the U. S. Department of Energy by the University of California, Lawrence Livermore National Laboratory under Contract No. W-7405-Eng-48. 


\section{Simulating shock-induced plasticity in single crystal $\mathrm{Cu}$}

\section{E.M. Bringa, P. Erhart, N. Tanushev, M. J. Caturla, B.D. Wirth(CMS) \\ D. Kalantar (NIF), and J. Stolken (NTED)}

Large scale molecular dynamics simulations with the embedded atom method (EAM) potentials are used to simulate shock propagation in single crystal $\mathrm{Cu}$ for different crystal orientations. Simulations were performed for a wide range of pressures (2-300 GPa) and agree well with recent experimental data. Large anisotropies are found for shock propagation in $\mathrm{Cu}$, with different plasticity mechanisms along different directions. We have observed for the first time the formation of nano-twins in shocked $\mathrm{Cu}$. Due to postshock evolution they could transform into the micro-twins seen in experiments at similar shock pressures. Experiments for "single crystals" deal with samples having a small initial defect density, including point defects, dislocations, etcetera. Therefore, simulations were also run for crystals with dislocation sources, vacancies and nano-void distributions before the shocks. Large vacancy concentrations (up to $0.5 \%$ ) do not change significantly the Hugoniot nor the Hugoniot elastic limit (HEL); however, the inclusion of voids decreases the HEL significantly. Simulations of the activation of a Frank-Read dislocation source also give insights into shock-induced dislocation multiplication process.

This work was performed under the auspices of the U. S. Department of Energy by the University of California, Lawrence Livermore National Laboratory under Contract No. W-7405-Eng-48. 


\title{
Using Segmental Isotopic Labeling and NMS Spectroscopy for Studying the Mechanism of Bacterial Transcription. A Potential Target for the Design of New Antibiotics
}

\author{
Julio A. Camarero', David. Fushman ${ }^{2}$ and James de Yoreo ${ }^{1}$ \\ ${ }^{1}$ Lawrence Livermore National Laboratory, University of California, Livermore, CA- \\ 94550, ${ }^{2}$ University of Maryland, College Park, MD-20742
}

Expressed Protein Ligation (EPL) is one of the most revolutionizing tools developed in the protein engineering field. This methodology allows the chemo-selective ligation of synthetic and/or recombinant polypeptides thus allowing the semi-synthesis of chemically modified proteins that can be used for multiple applications. One of such of applications is the use of EPL for obtaining segmental isotopic labeled proteins and therefore allows the use of Nuclear Magnetic Resonance (NMR) spectroscopy for the structural analysis of large proteins in solution. Selectively labeling an individual domain of a large multi-domain protein, overcomes the spectral complexity problems normally associated with big proteins $(>30-40 \mathrm{kDa})$, and allows the structural analysis of those labeled regions in the context of the full length protein. We are actually applying this methodology to study the molecular bases involved in the regulation of bacterial transcription. DNA-dependent RNA polymerases (RNAP) play a central role in transcription and therefore are a major regulatory target for gene expression. The regulation of prokaryotic transcription involves several protein-protein interactions between the different subunits of the RNAP complex as well as between several transcription factors. Characterization of these interactions, at the molecular level, is a prerequisite to fully understand transcription mechanisms and their regulation, which will help in the rational design of new antibiotics.

This work was performed under the auspices of the U.S. Department of Energy by the University of California, Lawrence Livermore National Laboratory under Contract No. W-7405-Eng-48. 


\section{Advances in High Sensitivity Portable LC/NMR \\ Sarah Chinn, Anthony Bernhardt, Vince Malba, Robert Maxwell}

It is well known that nuclear magnetic resonance (NMR) offers a non-destructive, powerful, structure-specific analytical method for the identification of suspect analytes. Integration of NMR methods with various separation techniques such as liquid chromatography (LC/NMR) enables detailed spectroscopic analysis of complex mixtures. An increasing need for field detection of chemical and biological agents warrants the development of a portable LC/NMR system. However, conventional NMR analysis is limited by sensitivity and resolution, resulting in the need for large sample volumes and extremely homogeneous, high magnetic fields produced by superconducting magnets. While recent advances in micro-receiver technology have demonstrated a $>70$-fold increase in detection limits, current portable NMR systems remain limited by magnetic field and resolution and are not compatible with other analytical techniques.

Progress towards a portable LC/NMR system at LLNL includes the development of highly sensitive radiofrequency microcoils using laser-lathe lithography and the construction of an NMR probe to be used with a 2 Tesla permanent magnet weighing only 1 kilogram. Because of the reduced sample size used in the microcoil, only a small region of magnetic field homogeneity is needed, thus eliminating the need for large superconducting magnets. Initial results obtained from this system will be presented along with future enhancements involving the incorporation of a compact LC system, smaller diameter microcoils, and a compact NMR electronics package. The application of portable LC/NMR technology to the detection of chemical and biological agents will be discussed. 


\title{
Assembly of oriented 2-dimensional protein and virus arrays by dip-pen nanolithography
}

\author{
Barry Cheung
}

\begin{abstract}
A general approach for the fabrication of oriented 2-dimensional protein and virus arrays has great potential to facilitate the determination of their structure by $\mathrm{x}$-ray diffraction and measurements of their surface potentials. We here present our ongoing effort towards the creation of nanoscale ordered templates with site-specific chemical linkers to proteins and viruses by dip-pen nanolithography. The orientations of these proteins and viruses can be controlled by selectively linking unique functional groups on their surfaces to specific chemical linkers on the templates. We will discuss preliminary results with viruses on patterned templates fabricated by micro-contact printing. We will also present future strategies integrated with microfluidics to promote ordering of protein arrays.
\end{abstract}

This work was performed under the auspices of the U. S. Department of Energy by the University of California, Lawrence Livermore National Laboratory under Contract No. W-7405-Eng-48. 


\title{
Structure and bonding of magnetocaloric materials $\mathbf{G d}_{5}\left(\mathrm{Si}_{\mathrm{x}} \mathbf{G e}_{1-\mathrm{x}}\right)_{4}$ and Related Systems
}

\author{
Wonyoung Choe
}

The unprecedented giant magnetocaloric effect (MCE) found in $\mathrm{Gd}_{5}\left(\mathrm{Si}_{\mathrm{x}} \mathrm{Ge}_{1-\mathrm{x}}\right)_{4}$, together with other exotic magnetic properties such as a colossal magnetostriction and a giant magnetoresistance, has initiated vigorous research activities on this and related $\mathrm{Ln}_{5}\left(\mathrm{Si}_{\mathrm{x}} \mathrm{Ge}_{1-\mathrm{x}}\right)_{4}$ systems ( $\mathrm{Ln}=$ lanthanide), because $\mathrm{Gd}_{5}\left(\mathrm{Si}_{\mathrm{x}} \mathrm{Ge}_{1-\mathrm{x}}\right)_{4}$ could be a promising candidate for near room temperature magnetic refrigeration and other applications. Large MCE response found in $\mathrm{Gd}_{5}\left(\mathrm{Si}_{2} \mathrm{Ge}_{2}\right)$ is closely related to a first-order magnetic phase transition between low temperature ferromagnetic, and high temperature paramagnic phase. This magnetic transition accompanies a simultaneous structural transition, which involves reversible covalent bond cleavage and formation by shear movement of $2 \mathrm{D}$ slabs in $\mathrm{Gd}_{5}\left(\mathrm{Si}_{2} \mathrm{Ge}_{2}\right)$. Interestingly, the three structures in $\mathrm{Gd}_{5}\left(\mathrm{Si}_{\mathrm{x}} \mathrm{Ge}_{1-\mathrm{x}}\right)_{4}$, i.e. $\mathrm{Sm}_{5} \mathrm{Ge}_{4}$, $\mathrm{Gd}_{5} \mathrm{Si}_{2} \mathrm{Ge}_{2}$, and $\mathrm{Gd}_{5} \mathrm{Si}_{4}$ type, are interchangeable by altering temperature, magnetic field, or $\mathrm{Si} / \mathrm{Ge}$ ratio.

To probe how the $\mathrm{Gd}_{5}\left(\mathrm{Si}_{\mathrm{x}} \mathrm{Ge}_{1-\mathrm{x}}\right)_{4}$ structures are affected by valence electron concentration, i.e., number of valence electrons per formula unit, a new series of compounds, $\mathrm{Gd}_{5}\left(\mathrm{Ga}_{\mathrm{x}} \mathrm{Ge}_{1-\mathrm{x}}\right)_{4}, \mathrm{x} \leq 2$, has been identified. Unlike $\mathrm{Gd}_{5} \mathrm{Si}_{2} \mathrm{Ge}_{2}$, which shows a room-temperature monoclinic structure, the $\mathrm{Gd}_{5}\left(\mathrm{Ga}_{\mathrm{x}} \mathrm{Ge}_{1-\mathrm{x}}\right)_{4}, \mathrm{x} \leq 2$ structures remain orthorhombic but show interatomic distances that are intermediate between $\mathrm{Gd}_{5} \mathrm{Si}_{4}$ and $\mathrm{Sm}_{5} \mathrm{Ge}_{4}$ type. Therefore, the control of shear movement between the slabs is possible by changing $\mathrm{Ga} / \mathrm{Ge}$ ratio. As part of this work, we have also constructed a "structure sorting map" based on ratios of crystallographic axes that will allow rapid assessment among the structures before detailed crystallographic studies are carried out. In addition to changing the valence electron concentration, we have also examined the evolution of these structures with the electronegativity differences between the two different minority components, i.e., $\mathrm{X}$ and $\mathrm{Y}$ in the generic formula $\operatorname{Ln}_{5}\left(\mathrm{X}_{\mathrm{x}} \mathrm{Y}_{1-\mathrm{x}}\right)_{4}$. Therefore, we have prepared various compositions of $\mathrm{Gd}_{5}(\mathrm{Ga}, \mathrm{Sb})_{4}$ as well and will present detailed structural characterizations and property measurements on this new series.

This work was performed under the auspices of the U. S. Department of Energy by the University of California, Lawrence Livermore National Laboratory under Contract No. W-7405-Eng-48. 


\title{
The Sol-gel Synthesis and Characterization of $\mathrm{Fe}_{2} \mathrm{O}_{3}-\mathrm{SiO}_{2}$ Nanocomposite Materials with a Large Iron Oxide Content
}

\author{
Brady J. Clapsaddle
}

Iron oxide-silica $\left(\mathrm{Fe}_{2} \mathrm{O}_{3}-\mathrm{SiO}_{2}\right)$ composite materials have previously been synthesized by a variety of methods, including sol-gel chemistry. Due to the slow condensation of the $\mathrm{Fe}_{2} \mathrm{O}_{3}$ precursors, the $\mathrm{Fe}_{2} \mathrm{O}_{3}$ content of these composite materials has been low, usually $<20 \mathrm{wt} \%$. Due to recent advances in our laboratory in the sol-gel synthesis of $\mathrm{Fe}_{2} \mathrm{O}_{3}$, several novel $\mathrm{Fe}_{2} \mathrm{O}_{3}-\mathrm{SiO}_{2}$ nanocomposite materials in which $\mathrm{Fe}_{2} \mathrm{O}_{3}$ is the major component have been synthesized. In a typical synthesis, the $\mathrm{Fe}_{2} \mathrm{O}_{3}$ precursor, $\mathrm{FeCl}_{3} \cdot 6 \mathrm{H}_{2} \mathrm{O}$, was mixed with a $\mathrm{SiO}_{2}$ precursor, tetramethoxy- or tetraethoxysilane, in ethanol and gelled using an organic epoxide. The $\mathrm{Fe}_{2} \mathrm{O}_{3}$ composition of the $\mathrm{Fe}_{2} \mathrm{O}_{3}-\mathrm{SiO}_{2}$ composite materials was varied from 55 to $97 \mathrm{wt} \%$ by adjusting the amount of siloxane added to the initial solution. Further processing of the resulting gels in supercritical $\mathrm{CO}_{2}(l)$ resulted in porous aerogel nanocomposite materials

with surface areas averaging $370 \mathrm{~m}^{2} / \mathrm{g}$. Several hybrid organic/inorganic nanocomposite materials, prepared using organically functionalized siloxanes as $\mathrm{SiO}_{\mathrm{x}}$ precursors, were also synthesized by this method. All of the composite materials prepared have been characterized by nitrogen adsorption/desorption methods, Fourier-transform infrared spectroscopy (FTIR), and transmission electron microscopy (TEM). In addition, energy filtered transmission electron microscopy (EFTEM) was used to show the uniform dispersion of both iron and silicon throughout the final material. The synthesis method presented is general for the synthesis of several other metal oxide- $\mathrm{SiO}_{2}$ nanocomposite materials, as well as other mixed metal oxide nanocomposites. Potential applications of the hybrid organic/inorganic nanocomposites as energetic materials will also be discussed.

This work was performed under the auspices of the U. S. Department of Energy by the University of California, Lawrence Livermore National Laboratory under Contract No. W-7405-Eng-48. 


\title{
Using Micro-Raman Spectroscopy for Rapid Identification of Bacterial Spores
}

\author{
Anthony Esposito, Chad Talley, Christopher Hollars, Thomas Huser (CMS) \\ Steve Lane (PAT)
}

\begin{abstract}
Raman spectroscopy is a valuable method for rapid, non-destructive chemical analysis. We have begun investigating the feasibility of using micro-Raman spectroscopy to identify single bacterial endospores. This method could provide a rapid alternative to current techniques involving cultivation of samples or antibody detection methods requiring large numbers of spores. We have obtained Raman spectra of individual spores from several species of the genus Bacillus, in order to determine if each species possesses a unique spectral signature. In addition, we have begun studies to determine if the detection of spores can be enhanced using surface-enhanced Raman spectroscopy (SERS). By greatly increasing the Raman scattering from the proteins on the spore coat exterior using SERS techniques, we are investigating if species-specific information can be obtained from the spore coat.
\end{abstract}

This work was performed under the auspices of the U. S. Department of Energy by the University of California, Lawrence Livermore National Laboratory under Contract No. W-7405-Eng-48. 


\section{Characterization of Nanostructural Features in Reactor Pressure Vessel Model Alloys}

Stephen C. Glade, B.D. Wirth, P. Asoka-Kumar, G. R. Odette*, and P.A. Sterne Lawrence Livermore National Laboratory

* University of California, Santa Barbara

The continued operation or lifetime extension of a number of nuclear power plants around the world requires an understanding of damage imparted to the pressure vessel steel by irradiation. This damage results in a high number density of nanometersized copper rich precipitates and sub-nanometer defect-solute clusters, which are the cause irradiation embrittlement. We present the results of positron annihilation spectroscopy (PAS) and small angle neutron scattering (SANS) characterization of the size, number density, and chemical composition of these nanometer sized features. PAS is uniquely suited for studies on these steels in that positrons preferentially localize in vacancies and in copper precipitates, identifying vacancies and vacancy clusters and providing local chemical environment information.

This work was performed under the auspices of the U. S. Department of Energy by the University of California, Lawrence Livermore National Laboratory under Contract No. W-7405-Eng-48. 


\title{
Development of an Enzyme-Based Photoluminescent Porous Silicon Detector
}

\author{
Bradley R. Hart ${ }^{1}$, Sonia E. Letant ${ }^{1}$, Staci R. Kane ${ }^{1}$, Masood Z. Hadi $^{2}$, Sharon J. Shields ${ }^{1}$, \\ and John G. Reynolds ${ }^{1}$ \\ ${ }^{1}$ University of California, Lawrence Livermore National Laboratory, Livermore, CA \\ 94551; ${ }^{2}$ Lockheed Martin Corporation, Sandia National, Laboratory, Livermore, CA \\ 94551
}

Photoluminescent (PL) silicon has been used for detection of organic substances. Devices made from this material utilize a reduction or increase of the PL of silicon caused by silicon interacting with specific chemicals. The specificity of the interaction depends upon the surface of the silicon, which can be modified through the Si-H sites. The goal has been to modify the surface to be attractive to a specific or related species. For example, porous silicon detectors have been attempted for penicillin detection by modifying the surface with penicillinase. Our attempts to extend this concept to BW and $\mathrm{CW}$ involve covalently modifying the hydride-terminated surface of porous silicon with an organic linker. This linker can then be used to attach various molecules to the surface including CW active enzymes. Surface modification is achieved by hydrosilation of various alkynes or alkenes, thereby providing reactive functionality. Conventional protein cross-linker chemistry is then used to extend the linker and provide for the attachment of various molecules. Characterization of these surfaces has been performed using IR and mass spectrometry techniques. In addition, examination of the PL spectra of the modified surfaces indicates that a high degree of photoluminescence is retained.

This work was performed under the auspices of the U. S. Department of Energy by the University of California, Lawrence Livermore National Laboratory under Contract No. W-7405-Eng-48. 


\title{
Synthesis of Nickel-Aluminide Foams
}

\author{
Andrea Hodge
}

Nickel-aluminide foams were synthesized from unalloyed nickel foams by using a two-step, high-activity pack-aluminizing process at 1273 and $1307 \mathrm{~K}$. After processing, the nickel aluminide foams exhibited the same structure as the original nickel foams (open-cells with hollows struts and low density). Single-phase NiAl foams, with average composition within $1 \mathrm{wt} . \%$ of stoichiometry and with $92 \%$ open porosity, were produced by first selecting the appropriate aluminizing time, and then annealing to homogenize the structure. Nickel wires and tubes were also aluminized at $1273 \mathrm{~K}$ and homogenized for various times to further investigate the aluminizing kinetics and the creation of Kirkendall pores. For aluminization depths up to about $100 \square \mathrm{m}$, Kirkendall pores can be avoided, leading to pore-free struts in the foam.

Foams with two different relative densities were tested under compression creep conditions for temperatures ranging from 1000 to $1373 \mathrm{~K}$, and stresses ranging from 0.1 to 1.3 MPa. For stress lower than 0.6 $\mathrm{MPa}$ the results follow power law creep while at higher stress power law breakdown was evident. In the former range, the creep exponent and the activation energy of the foams are 3.5 and $210 \mathrm{KJ} / \mathrm{mole}$, in good agreement with values reported for bulk NiAl.

This work was performed under the auspices of the U. S. Department of Energy by the University of California, Lawrence Livermore National Laboratory under Contract No. W-7405-Eng-48. 


\title{
Fate and Transport of Chemical Plumes in the Atmosphere
}

\author{
Tina Jayaweera
}

There are many types of chemicals released into the atmosphere either accidentally or on purpose. Examples include stack exhaust from energy plants, fumes from a toxic tire fire, or production by-products from a chemical plant. As the chemicals produced in the above examples can have an impact on human health, and, in the last case, be indicative in chemical warfare agent (CWA) production, it is important to know how the plume travels as well as how the chemicals in the plume evolve in the atmosphere. In conjunction with the Signatures group in NAI, we are currently primarily concerned with detecting the production of CWAs, by modeling the transport and chemistry of their potential production by-products. Currently, a code, written by researchers in the E\&E division that runs on the massively parallel ASCI machines, is able to accurately model the transport of a plume on a scale of a few city blocks. However, this code does not currently have the capability to model the chemical kinetics. A previous post-doc has developed a kinetic mechanism to model the combustion of sarin (GB), a main CWA; the current project is to adapt this mechanism to atmospheric conditions and to integrate it with the transport code.

This work was performed under the auspices of the U. S. Department of Energy by the University of California, Lawrence Livermore National Laboratory under Contract No. W-7405-Eng-48. 


\section{Smart membranes: filtration, immobilization and detection on a single chip}

Sonia E. Létant, Bradley R. Hart, Anthony W. H. van Buuren and Louis J. Terminello

A large variety of filtration membranes can be found on the market: pore diameters range from tens of nanometers to tens of microns, and various materials are available (ex: polycarbonate, polytetrafluoroethylene, cellulose esters). These products mainly act as passive devices, selecting molecules according to their size and some coarse chemical properties (ex: separation of hydrophilic and hydrophobic molecules).

The goal of our project is to build a bio-inorganic membrane which will be highly selective and 'smart'. The main idea is to etch pores in a silicon wafer to obtain a membrane and then to functionalize the pore walls with chemical anchors designed to specifically harvest target molecules. These membranes will be versatile since both pore size and pore wall functionality can be adjusted according to the target of interest. Our ultimate goal is to convert these passive membranes into 'smart' membranes, able to determine the concentration of target in situ and in real time. A way of doing this is to build a periodic array of pores and to use the membrane as a photonic waveguide. Other future applications like blood filtration and DNA detection will also be presented.

This work was performed under the auspices of the U. S. Department of Energy by the University of California, Lawrence Livermore National Laboratory under Contract No. W-7405-Eng-48. 


\title{
Modification of the Spin Polarization, Magnetic Properties and Electronic Structure of Thin Epitaxial Fe Films on GaAs(100) via the Introduction of Substrate Steps
}

\author{
Simon Morton
}

Epitaxial Fe thin films of up to $5.5 \mathrm{~nm}$ were deposited on singular and vicinal GaAs substrates and their magnetic and structural properties investigated by UPS, XPS, spin polarized photoelectron spectroscopy, magnetic linear dichroism in photoemission, VSM magnetization measurements and x-ray diffraction.

On both types of substrate the Fe grows predominantly as delta bcc-Fe. In agreement with literature results, the magnetization measurements and the magnetic linear dichroism results indicate very similar magnetic properties for the Fe films grown on the two substrate types with the exception of a substantially increased Curie temperature for the vicinal samples. Also, comparison of the spin polarized valence bands of the Fe films on the singular and the vicinal substrates reveal very significant differences in spin resolved band structure. The possible origins of these observations will be discussed.

Comparisons and rationalizations will also be made (were possible) between our observations on $\mathrm{Fe}$ on GaAs and literature reports for other $\mathrm{Fe}$ thin film systems. For example, we have found that the spin polarized valence bands of Fe deposited on the vicinal GaAs exhibit similar features to those reported in the literature for $10 \mathrm{~nm} F(100)$ films on $\mathrm{Cu} 3 \mathrm{Au}(100)$. In contrast, the spin polarized valence bands of Fe films on the singular GaAs are very different to all $\mathrm{Fe}$ thin film literature reports, with the differences concentrated in the minority spin channel.

This work was performed under the auspices of the U. S. Department of Energy by the University of California, Lawrence Livermore National Laboratory under Contract No. W-7405-Eng-48. 


\title{
Multi-Scale Structural Study of the $\delta \rightarrow \alpha^{\prime}$ Transformation in a Pu-Ga Alloy
}

\author{
Erik Nelson, Kerri Blobaum, Mark Wall, Patrick Allen, Adam Schwartz
}

Optical microscopy and extended x-ray absorption fine structure spectroscopy (EXAFS) were used to investigate changes in the grain morphology and in the local atomic environment of plutonium and gallium atoms through the $\delta \rightarrow \alpha^{\prime}$ martensitic transformation in a Pu-Ga alloy. Prior to the EXAFS experiments, a 2 at $\%$ Ga-doped $\delta$ $\mathrm{Pu}$ sample was quenched into the upper "nose" of the time-temperature-transformation (TTT) curve to form $\alpha^{\prime}-\mathrm{Pu}$, while a second identical sample was left untransformed. Optical microscopy revealed $\alpha^{\prime}$ particles present in the quenched sample. EXAFS was used to compare the structural behavior of the pre-transformed and untransformed samples upon cooling below the transition temperature. EXAFS spectral components attributed to both $\alpha^{\prime}-\mathrm{Pu}$ and $\delta$-Pu were observed in the pre-transformed sample, and the $\mathrm{Pu}$ and $\mathrm{Ga}$ EXAFS spectra indicate similar local atomic environments, suggesting $\mathrm{Ga}$ substitution for $\mathrm{Pu}$ atoms in both the $\alpha^{\prime}-\mathrm{Pu}$ and $\delta$-Pu structures.

This work was performed under the auspices of the U. S. Department of Energy by the University of California, Lawrence Livermore National Laboratory under Contract No. W-7405-Eng-48. 


\title{
Simulating Nucleation in Irradiated Materials and Aerosols
}

\author{
J. B. Sturgeon, M. P. Surh and W. G. Wolfer \\ Lawrence Livermore National Laboratory, Livermore, CA 95550
}

The effectiveness of structural components in nuclear reactors is dependent in large part upon their resistance to void swelling. Here we present a stochastic treatment of vacancy cluster formation in irradiated 316-stainless steel. The cluster distribution function is evolved using a two-step treatment that first evolves the monomer concentration analytically from rate-equations that are coupled to the void-distribution. Secondly, linearized void-monomer reaction rate equations are used to evolve the voiddistribution. By incorporating a Fokker-Plank continuum description with a Monte Carlo implementation, this model has been extended to allow void growth to very large sizes. Void nucleation proceeds in this model despite the lack of helium or other gas impurities and in the absence of any production bias. However, the intrinsic bias of dislocations for preferential interstitial capture and its evolution with the changing microstructure is essential to predict the onset of void swelling. Our new implementation runs to long times and can easily be extended to other materials. The results from our simulations are in reasonable agreement with experimental values of steady-state swelling and the terminal void number density.

We have recently adapted this method to develop a dynamic aerosol nucleation code which enables us to simulate the evolution of the particle size distributions for aerosols. Currently we are studying the aggregation dynamics of water molecules to droplets in air at ambient conditions.

This work was performed under the auspices of the U. S. Department of Energy by the University of California, Lawrence Livermore National Laboratory under Contract No. W-7405-Eng-48. 


\title{
Sampling and Analysis of Bacterial Spores and Vegetative Bacterial Cells Using Real-Time Bio-Aerosol Mass Spectrometry
}

\author{
Herbert J. Tobias, David P. Fergenson, Matthias Frank, and Eric E. Gard, \\ Chemistry and Material Science Directorate, Lawrence Livermore National Laboratory, \\ Livermore, California 94550.
}

The inlet system of a real-time single-particle mass spectrometric technique called Bio-Aerosol Mass Spectrometry (BAMS) was developed in order to efficiently screen and identify bio-aerosols of public health concern.

Aerosol nozzles used in many single-particle analysis systems efficiently focus particles that have aerodynamic diameters in a very narrow size range (i.e. around 1 micron). The few systems that recently incorporated aerodynamic lenses for better particle transmission to the analyzer region are designed to efficiently focus particles with diameters in the sub-micron range, which are of interest in the study of atmospheric aerosols and fundamental aerosol formation processes. However, effective analysis of bio-aerosols, such as spores and vegetative bacterial cells, requires efficient sampling of particles with diameters in the super-micron range. Therefore, aerodynamic lenses that focus a broader range of larger particles ( 1-5 microns in diameter) were designed to be used as the inlet system for an aerosol time-of-flight mass spectrometer.

Here, polysytrene latex spheres of different particle diameters that represent the aerodynamic size range of bacterial spores and cells are used to evaluate the focusing efficiency of the new lens system and compare it with the standard inlet nozzle. The focused particles are efficiently sampled into a differentially-pumped vacuum system, where they are individually sized by the time delay measured between two scattering lasers when a single particle passes through them. The particles are then individually analyzed for their chemical composition using dual-polarity laser desorption ionization time-of-flight mass spectrometry. The technique should result in improved sampling efficiency and analysis of bacterial aerosols, which normally range in size from 1-10 microns in diameter.

This work was performed under the auspices of the U. S. Department of Energy by the University of California, Lawrence Livermore National Laboratory under Contract No. W-7405-Eng-48. 


\title{
Electronic and Structural Responses of Materials to Ultashort and Intense Laser Pulses
}

\author{
B. R. Torralva and P. E. A. Turchi \\ Chemistry and Materials Science \\ P.O. Box 808, L-353 \\ Lawrence Livermore National Laboratory \\ Livermore, CA 94551
}

The study of the interaction of matter with ultrashort and intense laser pulses is still a frontier of science. The ability to explore this new regime has led, and continues to lead, to new discoveries. The usual approximations of theoretical physics and chemistry break down under these conditions, and the multi-scale nature of the electron and ion dynamics makes this a challenging computational problem. Here, we use a recently developed nonadiabatic quantum molecular dynamics model to investigate the interaction of ultrashort laser pulses with solids and molecules. Results will be presented for the photoexcitation of coherent phonons in bulk and surface group IV and III-V semiconductors, elucidating the differences between resonant and non-resonant excitations; the photofragmentation of fullerene $\mathrm{C}_{60}$ and a $\mathrm{C}_{10}$ linear chain; the photoinduced $2+2$ cycloaddition reaction; as well as the selective photodissociation reaction of cyclobutane, revealing the formation of the tetramethylene diradical reaction intermediate. Comparison with available experimental results will be made.

This work was performed under the auspices of the U. S. Department of Energy by the University of California, Lawrence Livermore National Laboratory under Contract No. W-7405-ENG-48. Partial support from NATO under Contract No. CRG 941028 is gratefully acknowledged. 


\title{
Formation of GaAs Nanocrystalline Films by Femtosecond Pulsed Laser Deposition
}

\author{
T. W. Trelenberg, L. N. Dinh, and M. Balooch
}

The properties of femtosecond pulsed laser deposited GaAs nanoclusters were investigated. Nanoclusters of GaAs were produced by laser ablating a single crystal GaAs target in vacuum or a buffer gas using a Ti-Sapphire laser with a 150 fs minimum pulse length. For in-vacuum deposition, XRD, AFM, and SEM showed that the average clusters were about $7 \mathrm{~nm}$ for laser pulse-lengths between $150 \mathrm{fs}$ to $25 \mathrm{ps}$. The cluster size dropped to about $1.5 \mathrm{~nm}$ at a pulse-length of 500ps and it was also observed that film thickness decreased with increasing laser pulse length. A reflective coating, which built up on the laser admission window during ablation, reduced the amount of laser energy reaching the target for subsequent laser shots and developed more rapidly at longer pulselengths. This observation indicates that non-stoichiometric (metallic) ablatants were produced more readily at longer pulse-lengths. The angular distribution of ejected material about the target's normal was well fitted to a bi-cosine distribution of $\cos ^{47} \theta+$ $\cos ^{4} \theta$ for ablation in vacuum. These GaAs nanocluster films were found by XPS and AES to be non-stoichiometric, containing excess Ga or As. However, stoichiometric GaAs nanocrystals were produced when the ablation was carried out in the presence of a buffer gas with a pressure in excess of $50 \mathrm{mTorr}$. At buffer gas pressure on the order of 1 Torr, it was found that the stoichiometry of the ablated target was also preserved. Our experiments indicate that both laser pulse length and buffer gas pressure play important roles in the formation of multi-element nanocrystals by laser ablation. The effects of gas pressure on the target's morphology and the size of the GaAs nanocrystals formed will also be presented--

This work was performed under the auspices of the U. S. Department of Energy by the University of California Lawrence Livermore National Laboratory under Contract No. W-7405-ENG-48. 


\title{
Fabrication of luminescent nanostructures by dip-pen nanolithography
}

\author{
Brandon Weeks
}

We used a combination of dip-pen nanolithography and scanning optical confocal microscopy to fabricate and visualize luminescent nanoscale patterns of various materials on glass substrates. We show that this method can be used successfully to push the limits of dip-pen nanolithography down to controlled deposition of single molecules. We also demonstrate that this method is able to create and visualize protein patterns on surfaces. Finally, we show that our method can be used to fabricate polymer nanowires of controlled size using conductive polymers. We also present a kinetic model that accurately describes the deposition process.

This work was performed under the auspices of the U. S. Department of Energy by the University of California, Lawrence Livermore National Laboratory under Contract No. W-7405-ENG-48. 


\title{
Atomic-Resolution Transmission Electron Microscopy of the Intergranular Structure of a $\mathrm{Y}_{2} \mathrm{O}_{3}$-Silicon Nitride Ceramic
}

\author{
A. Ziegler ${ }^{1}$, C. Kisielowski ${ }^{2}$, M. J. Hoffmann ${ }^{3}$, and R. O. Ritchie ${ }^{1}$ \\ ${ }^{1}$ Materials Sciences Division, Lawrence Berkeley National Laboratory, and Department \\ of Materials Science \& Engineering, University of California, Berkeley, CA 94720, USA \\ ${ }^{2}$ National Center for Electron Microscopy, Lawrence Berkeley National Laboratory, \\ Berkeley, CA 94720, USA \\ ${ }^{3}$ Institut für Keramik im Maschinenbau, Universität Karlsruhe, D-76131 Karlsruhe, \\ Germany
}

High-resolution transmission electron microscopy (HRTEM) employing focusvariation phase-reconstruction methods is used to image the atomic structure of grain boundaries in a silicon nitride ceramic at a resolution of $0.8 \AA$. Complementary energydispersive X-ray emission spectroscopy experiments revealed the presence of yttrium ions segregated to the $0.5-0.7 \mathrm{~nm}$ thin amorphous boundary layers that separate individual grains. Our objective here is to discern whether the yttrium ions attach to the prismatic planes of the $\mathrm{Si}_{3} \mathrm{~N}_{4}$ at the interface towards the amorphous layer, using Scherzer and phase-reconstruction imaging, as well as image simulation. Although crystal structure images from thin $(<7 \mathrm{~nm})$ boundary area areas, do not reveal the attachment of yttrium at these positions, lattice images from thicker areas do suggest the presence of yttrium at these sites. It is concluded that most of the yttrium atoms are located in the amorphous phase and only few atoms are attached to the prism plane of the matrix-grain/grain-boundary interface; the line concentrations of such yttrium in the latter location are estimated to be one yttrium atom every $16.8 \AA$.

This work was performed under the auspices of the U. S. Department of Energy by the University of California, Lawrence Livermore National Laboratory under Contract No. W-7405-ENG-48. 